\title{
Do You Speak, Write and Think in Informatics?
}

\section{Mária Csernoch, Erzsébet Dani}

University of Debrecen, Faculty of Informatics, Kassai út 26, 4028 Debrecen, Hungary, csernoch.maria@inf.unideb.hu

University of Debrecen, Faculty of Humanities, Egyetem tér 1, 4032 Debrecen, Hungary, dani.erzsebet@arts.unideb.hu

\begin{abstract}
The dissemination of research results might be as crucial as the research itself. The widely accepted two major forms of dissemination are written papers and live presentations. On the surface, if we see the problem in hyper attention mode, these documents are different in nature. However, their preparation requires the same problemsolving approach, and beyond that, they share the fundamental rules of design since both are extended text documents, with varying proportions of text and/or non-text contents and static and/or dynamic media types. Closely related to this problem is the phenomenon of different types of attention (hyper and deep attention), thinking (fast and slow) modes, and problem-solving approaches (high- and low-mathability). In the world of immense and various forms of data and information, the role of so-called hyper attention is fundamental and inevitable, but the presence of deep attention is essential as well. In the present paper the knowledge items involved and shared in the design and the preparation of text-based documents are detailed from the view of concept-based problem-solving, the perspective of attention and thinking modes, along with samples originated from various sources and subject matters. The aims are to discuss the theoretical background of effective and efficient document design and preparation and to call attention to the consequences of ignoring, neglecting the proper use of attention types and thinking modes.
\end{abstract}

Keywords: end-user computing; attention modes; thinking modes; deep and surface computer problem-solving approaches; low- and high-mathability

\section{Introduction}

\subsection{Who is an End-User and what Problems He/She is Faced with?}

One of the most common scenarios is when an end-user is clicking around in computer applications suffering, but still claiming that - "I can do it, that's enough." - "No, that's not," - is the short reflection to this frequently repeated and 
widely accepted but low-mathability [1] [2] [3] slogan. The long explanation should cover all the efficient and effective problem-solving approaches accepted and practiced in other sciences, but hardly recognized and applied in end-user informatics and problem-solving.

By definition, "An end-user is the person that a software program or hardware device is designed for. The term is based on the idea that the 'end goal' of a software or hardware product is to be useful to the consumer. To simplify, the end-user is the person who uses the software or hardware after it has been fully developed, marketed, and installed" [4], in short, "the ultimate consumer of a finished product" [5].

However, problems arise when the complexity, the requirements, the set-up of a product is far beyond the scope of the target population. Further difficulties are faced when not one but a whole set of products are required to be handled. The figure becomes even more frightening when long forgotten or never learned knowledge, skills, abilities, attitudes ${ }^{1}$ should be brought into these computational environments which effectiveness is clearly proved in high-mathability studies [8] [9] [14]. In general, when the right proportion of fast and slow thinking [6] should be activated for effective and efficient problem-solving, leaving low-mathability [3] methods behind. In the context of the attention issue: difficulties arise when the end-user needs to switch from hyper to deep attention [7].

\subsection{The Real End-User}

By experience, an end-user is the person who has never been educated in informatics/computer sciences (I/CS) or was taught how to navigate interfaces or self-taught or an I/CS professional but neglects or ignores non-professional informatics. A person who can be persuaded by software companies that they are in continuous need of newer versions and they do not need to know anything for using these applications, so they are the perfect ignorant targets.

This issue goes even further when experts are aware of end-users' erroneous spreadsheet documents causing serious financial losses and search for explanations. In his research, Panko concluded that the behavior of end-users can be explained by their bad habit of thinking [10]-[12]. However, our research in computer problem-solving approaches, attention modes [7] [14], thinking modes [6], and mathability reveal that thinking is not bad, but applied in incorrect, nonsuitable situations, primarily focusing on low-mathability approaches in hyper attention mode.

1 Closely related to this issue is the topic of digital literacy and competence, because not even the new generations are born with digital skills which need to be consciously acquired and continuously developed, similar to mother tongue language skills. 


\subsection{The Problem-Solving Approaches of End-Users}

The typology of computer problem-solving approaches [17] [18] and levels of understanding [19] [20], built upon traditional sciences with amendments matching the peculiarities of computers, was created for classifying computerrelated activities (Figure 1). This typology consists of two hypernyms - deep and surface approaches - and five hyponyms, two and three, respectively.

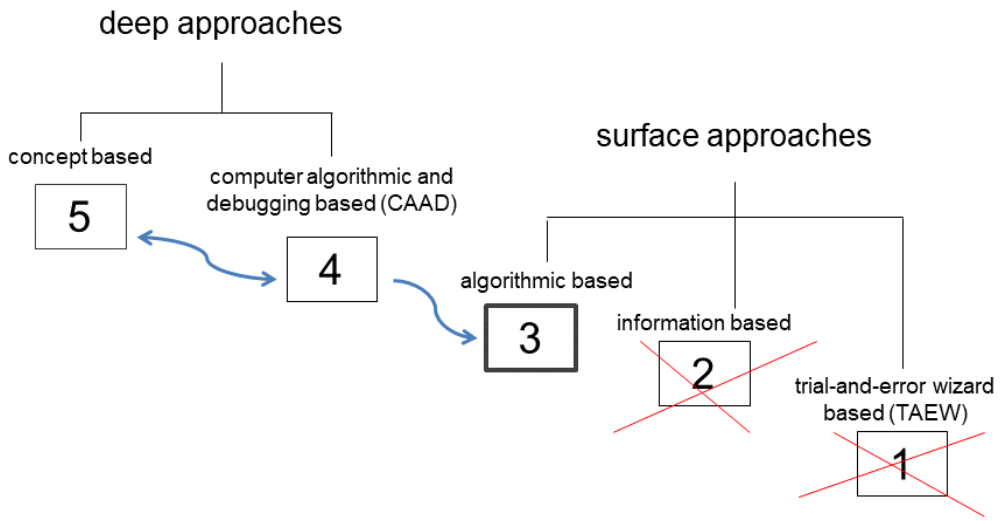

Figure 1

The typology of computer problem-solving approaches [17]

Considering the thinking modes - fast and slow [6] -, at Level 3 fast thinking, while at the other levels slow thinking work (Figure 1). With the arrows, the primary directions of communication between the different levels are indicated. There is continuous communication between Levels 5 and 4, where/when the first two steps of problem-solving take place. Level 4 is the position where schemaconstruction takes place, and these schemata are called/retrieved at Level 3. However, we must be aware that any backtracking is possible when errors or hiatus are recognized (these arrows are not included in Figure 1). Approaches at Levels 2 and 1 can hardly be considered problem-solving since they are strongly connected to the surfaces, they are interface navigation in hyper attention mode.

Considering the different computer problem-solving approaches and the documents handled - created and modified - by end-users clearly reveal that the experienced description of the end-user (Section 1.2) is more appropriate than the definition (Section 1.1). On the one hand, end-users are not able to function, as they should according to the definition. On the other hand, their productivity, effectiveness is much lower than excepted [10]-[12] [22]. Consequently, they perfectly fulfill the good customer image of software companies but lack computational thinking skills [21]. 
In the present paper, the speaking-writing-thinking approach of end-user computing is detailed, compared to the widely accepted surface approach, lowmathability erroneous methods (Figure 1). Furthermore, the essence of the concept-based problem-solving [23] - proved high-mathability methods [3] supported by the appropriate proportion of attention [6] and thinking modes [13] is drawn, confronted with samples of erroneous practice [18] [22].

\subsection{The Attention-Phase Perspective of the Typology}

The problem raised in the present paper connects closely with the HY-DE (HYper and DEep attention) model at this point. The model was created by Erzsébet Dani [7] for the true consumers of the new technologies and applications, for the "bitgenerations" (generations Y and Z). Since the basic unit of computer data is the bit, the children of the computer age can be identified with this denomination. The world of digital data produces the phenomenon of hyper attention, as described by Katherine N. Hayles [13]: "switching focuses rapidly among different tasks, preferring multiple information streams, seeking a high level of stimulation, and having a low tolerance for boredom". Based on these characteristics, without any proof, Prensky's concept of digital natives and immigrants indicates that there is no need for deep attention-driven activities in the digital era [24]. However, the pattern is not that simple -in contrast to Prensky [24] -, Kirschner \& De Bruyckere [25] proved that digital native and multitasking is only a myth.

It must be noted, though, that surface or hyper-attention approaches have not only negative but also positive aspects. In fact, it is necessary for processing data in the world of the information superhighway. However, deep attention is also needed, because in this attention phase the focus is on one issue, which leads toward deeper, constructive thinking and the resolution of the recognized and understood problems [25].

The basic concept behind the model is the shifting pattern of the two forms of attention: hyper and deep attention. The main goal is to influence and control the hyper attention phase consciously in order to keep it in the background and effect a shift that activates the needed deep attention. The phase-change is connected by a mixed attention section, in which both attention states are present, but with reversed intensity (i.e., decreasing hyper attention and increasing deep attention).

The original theoretical model has two stages in the teaching-learning process: one for the instructor and one for the student. The forms of attention tend in opposite directions. In the instructor stage, attention-training moves from hyper to deep attention, with instructional dominance, while in the independent-student-activity seminar-stage the movement is from deep to hyper attention, with decreasing teacher activity [7]. 
The HY-DE philosophy and attention-training strategy have a place in the typology and strategic arsenal of computer problem-solving approaches. And, if it is there, we have the possibility to influence the shift between the forms of attention.

\subsection{Concept-based Problem-Solving: a Dream in Computer Problem-Solving}

In 1954 György Pólya published his well-known book entitled "How To Solve It. A New Aspect of Mathematical Method." [23], and has been proved effective and efficient in the meantime in various subjects and sciences. In his book, Pólya set up four steps of real-world problem-solving, which are summarized as follows:

- First. Understanding the problem.

- Second. Devising a plan. Find the connection between data and the unknown. You may be obliged to consider auxiliary problems if an immediate connection cannot be found. You should obtain eventually a plan of the solution.

- Third. Carrying out the plan.

- Fourth. Looking back. Examine the solution obtained. (pp. xvi-xvii)

According to Pólya's problem-solving approach, the first step is the understanding of the problem. In the present frame, this is the phase when the original real-world problem must be broken up, and a new, a more abstract structure must be built, which is relevant to the interface where the solution is meant to be carried out. This is the first, speaking session in the process of problem-solving, where we must take notes, draw figures, connections, introduce notations, using as far as possible the terminology of both the subject matter and the solution-interface.

In the second phase, the planning takes place. Primarily, we must consider what previously built schemata can be applied and what novel items are involved and must be built. After setting up the places and the propositions of old and new knowledge items, the execution order of the solution process must be decided. This is the design session of problem-solving in our model.

The third step is the actual solution when the plan is carried out. In end-user problem-solving, the content must be presented in a form of a document, and following that commands must be carried out according to the plan. This is the writing phase of our model: creating and formatting the content, adding visual data, creating formulas, etc.

The fourth step is discussing/debugging. In this phase strictly, but not exclusively, the result must be evaluated: we must check whether the solution is correct or not. Furthermore, we must take into consideration whether we can find other solutions, and finally, the possibility of abstraction. This is the second speaking phase, when all the results must be checked, contrasted to our expectations, more effective and 
generalized solutions must be looked for. This implies more than using a concept; it involves the ability to select an appropriate approach from understood alternatives [19] [26]. The generalization of end-user solutions plays a crucial role in the problem-solving process since these documents are meant to substitute hardcoded, paper-based, typed, or handwritten documents. Considering this purpose, the major characteristic of the digital documents is changeability, modifiability. This intention is well served with the definition of correctly edited and formatted spreadsheet [10]-[12] [28] [29] and text documents [18].

In general, the concept-based problem-solving approach is a slow thinking process that requires both hyper and deep attention modes. At this point, the question arises, how a slow thinking method can be integrated into a rapidly changing digital world. We argue in Chapter 1.6 that instead of the widely accepted, exclusively utilized hyper attention mode, the combination of hyper and deep attention modes must be introduced, where fast thinking can come into action with a firm knowledge background, built up in slow thinking processes.

\subsection{Concept-based Problem-Solving: the Reality}

Panko, considering spreadsheets, claimed that "thinking is bad" [12]. His paper reveals that working on spreadsheet problems, end-users apply slow thinking, in other words, they engage surface-approach methods, creating erroneous documents of various types [10]-[12] [22]. He claims in order to avoid errors, fast thinking (so the presence of schemata [26] are emphasized here) should be applied, which is one of the major characteristics of experts [6] [30] [31]. At this point, we beg to disagree with Panko's thoughts to some extent. As mentioned above, thinking slow is not bad, when it is applied in the right situation. In fact, thinking slowly, that is, working in deep attention phase is essential in the process of learning and creating. By carefully reading Panko's paper, it was revealed that he found that only Levels 1 and 2 - surface approach navigation methods - are applied in spreadsheet activities (Figure 1). These two approaches are

- the information based - practiced interface management and

- the trial-and-error wizard-based - interface browsing.

They require slow thinking, but are not problem-solving, consequently, do not provide reliable solutions and results. When comparing Panko's finding and the four steps of Pólya's problem-solving, we can conclude that only the third step is activated, the others are ignored, which methods lead to surface navigation in hyper attention mode.

Similar patterns can be recognized in text management, in the two major textbased, end-user-handled document types:

- text documents - prepared with word processors and

- presentations [18]. 
End-users author, modify, disseminate a huge number of erroneous documents, without recognizing their mistakes, errors. An analysis of the documents reveals that almost exclusively, activities at Levels 1 and 2 - surface approach navigation methods - are carried out, strictly in the hyper attention phase. Ben-Ari entitled this approach as bricolage [32], which expression clearly demonstrates end-users' incomprehensibility of the matter and their non-existing problem-solving strategies.

In general, it was found that end-user computing lost or never has found or never wanted to find the four steps of Pólya's problem-solving approach. Only, the third step exists with all its consequences:

- High demand on human and computer resources - authors' time spent on problems, the number of agents participating in solving a particular problem, reader's and audience's difficulty of understanding, not allowing space for hyper attention.

- Erroneous solutions and results causing financial losses [10]-[12] [22] [28] [29] not claiming to deep attention.

- End-users being ignorant of their ignorance - over-confidence and not rarely arrogance, proudly disseminating erroneous documents [33]-, hyper attention is used in inappropriate situations.

- The reproductivity of bricolage due to the lack of well-qualified teachers [34] and teaching materials [18] [32], due to the myths of the digital natives [25], unproved and disseminated statements, based on only hyper attention practice [34].

\section{Presenting Errors}

\subsection{Utilizing Erroneous Documents}

As detailed, erroneous documents have flooded both the private and the business spheres as a consequence of the shift from a textual universe to the digital world. To worsen the case, most of them are uploaded on the Internet, available for anyone, presenting samples and allowing their faster reproduction. The question is how we can stop and/or reverse this undesired progress. One solution is education, where these authentic sources can serve as practicing materials to demonstrate the errors and their consequences. The method is borrowed from other sciences and teaching programming languages. Especially, in the latter, erroneous programs are frequently used in introductory programming classes to lighten the burden of novices writing programs and to prepare them for debugging throughout their lives. Another possibility of calling attention to the problem is presenting at conferences that are open to such challenges. 
The advantage of using authentic erroneous documents in education is, beyond providing inexhaustible sources, their effect on students. The force of authenticity plays a crucial role. First, the shock when the original authors are recognized, not rarely highly qualified persons, in publicly available materials and later on the proudness of "I-can-do-better". Finally, we cannot leave out the motivating aspect of the content. Any subject can be found which students are interested in, matches their age, gender, their background knowledge, and the aims of their classes.

In spite of the numerous advantages of using authentic erroneous documents for educational purposes, we can hardly find examples of good practices. As mentioned, in end-user computing and teaching end-user computing, almost exclusively low-mathability approaches are present, which focus on tools in hyper attention and slow thinking modes, without any thought of preparing and presenting real-world problems in classes. Consequently, the didactics of this approach is underrepresented either in scientific discussions or in the everyday classroom experience.

\subsection{Collecting Erroneous Documents}

\subsubsection{Finding Keywords}

One of the major characteristics of concept-based problem-solving and teaching is that authentic or adequate problems must be presented and solved. The major source of erroneous documents is the Internet, where millions of documents are available with the agreement of the authors by the act of uploading them.

The first concern of finding erroneous documents on the Internet is how to convince browsers to find and present only them. Teaching experience proves that quite often students and even parents find this task unsolvable, arguing that they typed in the search field "erroneous Word document" and its numerous variations, but no matches came along.

The problem with the "erroneous Word document" or "erroneous presentation" as keyword is that this search is not planned. Again, the first two steps of conceptbased problem solving is ignored and end-users mindlessly jumped into the third step: "executing". In this case, the search is carried out in hyper attention mode. By analyzing the task - switching to deep attention mode -, it should become obvious that the keyword "erroneous" would not provide results. Authors do not upload erroneous documents intentionally for at least two reasons: (1) nonfinished works are usually not uploaded, especially not with the title "erroneous", and (2) authors are not aware of their errors, consequently, they cannot express explicitly this feature of their documents. 


\subsubsection{Searching for the Suitable File Types}

The second concern of this search is the type of document. Again, teaching experience proved, that searching for a specific type is not obvious and practiced, in spite of its frequency. In most of the browsers by default, the searching of the whole Internet is offered (Figure 2), regardless of file types, and only after launching the first search, special search is available. However, these offers are rather media-type and not file-type orientated. This setup of browsers leads to the misconception - built up again, on hyper attention activities and practice - that typing the extension into the search field would provide the desired document formats (Figure 3).

\section{$G$ Search the Web}

Figure 2

The default search option on launching a browser

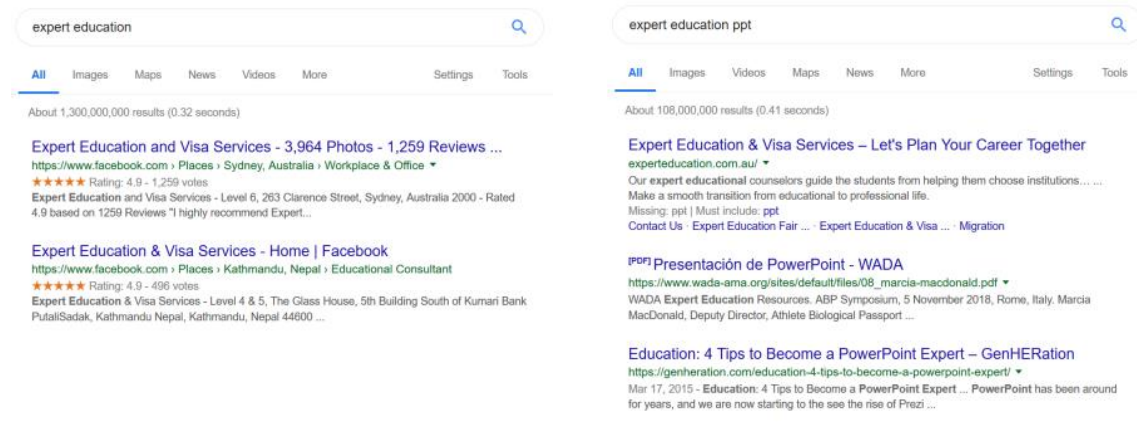

Figure 3

A preliminary search in the selected subject (left) and its extended search based on the misconception that special file types are available by typing the file extension into the search field (right)

any format
Autodesk DWF (.dwf)
Google Earth KML (.kml)
Google Earth KMZ (.kmz)
Microsoft Excel (.xls)
Microsoft PowerPoint (.ppt)
Microsoft Word (.doc)
Rich Text Format (.rt)
Shockwave Flash (.swf)

Figure 4

An extract of the file types offered by Google Advanced search 

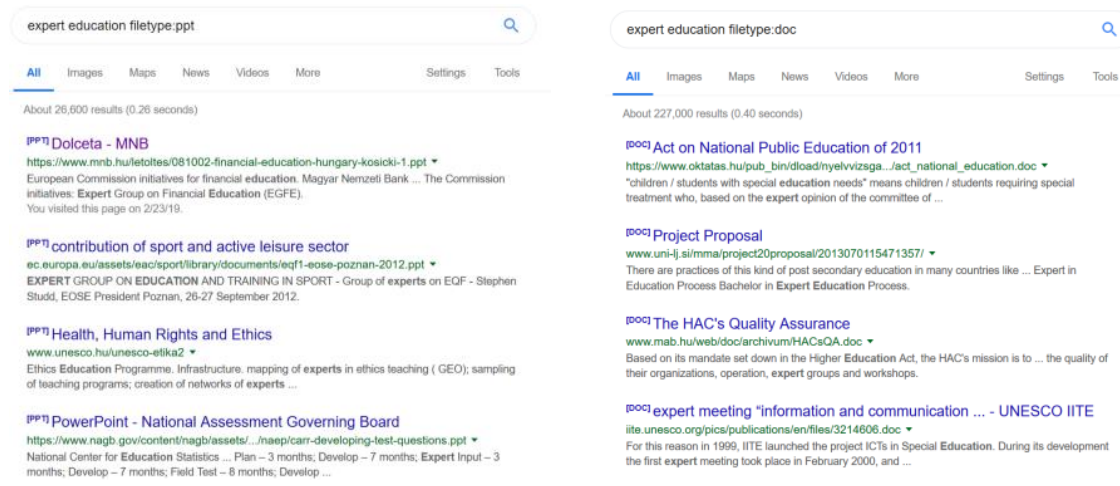

Figure 5

Searching for old PowerPoint and Word documents, with the extension of ppt and doc, respectively

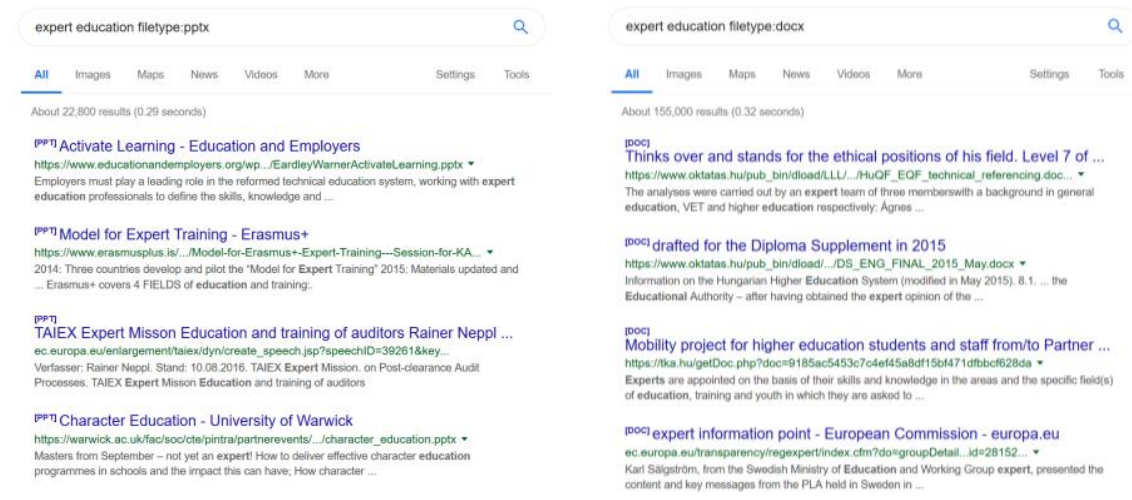

Figure 6

Searching for newer PowerPoint and Word documents, with the extension of pptx and docx, respectively

As mentioned, the content - the subject of the search - must be clarified in the first step of the problem-solving process (Figure 5, Figure 6), in the present case, the error types that we are looking for. Without conscious and meticulous design of the content, the search will lead to endless browsing without any result. Being aware of the teaching or research goals the task seems feasible.

After all these technical details and thousands of listed documents, comes the discussion phase of problem-solving, considering the content. The discussion requires both hyper and deep attention mode, where tens or hundreds of documents must be analyzed to find the document(s) which match our requirements the best. At this point of the discussion phase, we must realize that not finding the exact match is not because there are no errors in the documents, but the other way around, they contain so many errors that finding only the specified one is challenging (for details on error classification see [18]). 
In education, the students' mental development, background knowledge, age, the field of interest, the aims of the classes, etc. must be taken into consideration when a document search is launched.

\section{Designing Documents}

One of the most demanding subjects of end-user computing is the handling of design-based styles (Figure 7, Figure 8).

\subsection{Headings as Built-in Styles}
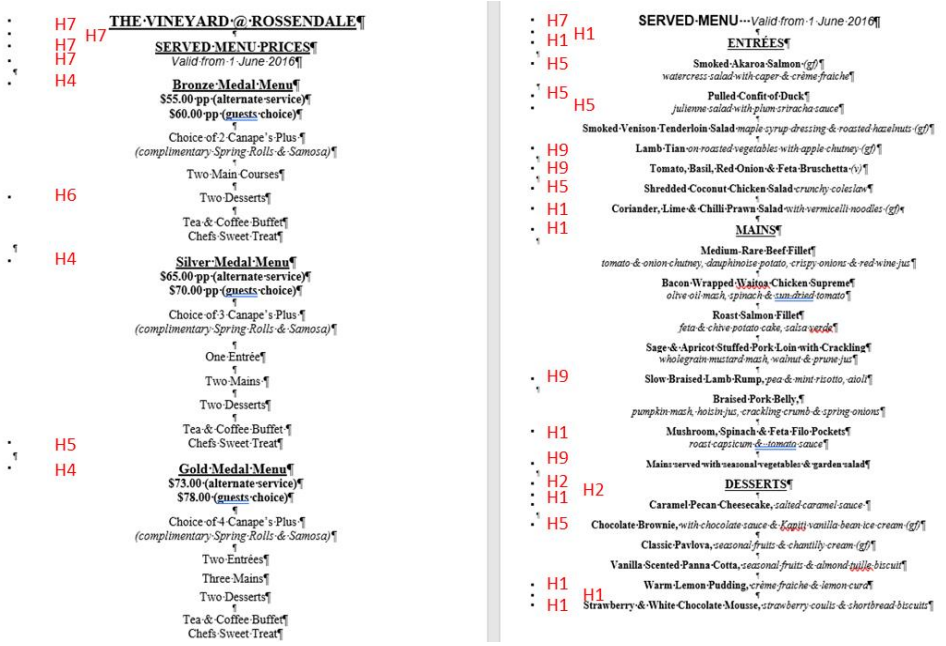

Figure 7

An incorrect style-combination is applied to a document. The red letter-number combinations indicate the header styles. The indented marks indicate those styles which are applied to empty paragraphs ${ }^{2}$.

Software companies are rather known for selling their products by understating their complexity and advertising the user-friendly approach. Even though these programs cannot avoid offering design tools for helping uneducated users, the question is how end-users can apply these features, how they make the best of these offers. In this paper, not the different styles and their quality are our concern, but how end-users can apply effectively the built-in styles, nonetheless their own?

2 na “THE VINEYARD @ ROSSENDALE”. Retrieved February 24, 2019. from https://www.rossendale.co.nz/?wpdmact=process\&did=MjUuaG90bGluaw==. $\quad$ A4 SERVED MENU. inc prices jun 2016.doc. 
Microsoft Word offers several levels of headings guiding and inviting end-users for their use. "Headings make text stand out and help people scan your document. ... The simplest way to add headings is with heading styles. Using heading styles means you can also quickly build a table of contents, reorganize your document, and reformat its design without having to manually change each heading's text." [36].

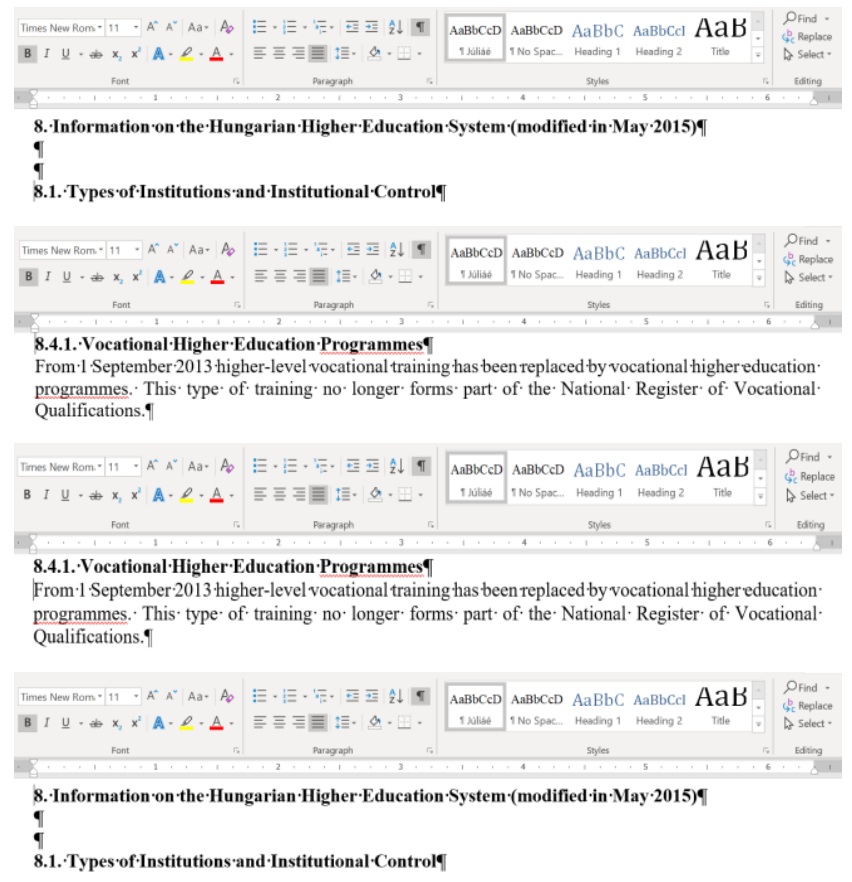

Figure 8

Incorrect use of costume style that does not allow the creation of an automated Table of Contents ${ }^{3}$

In Figure 7 a completely inconstant use of heading styles is presented. First, the styles are applied arbitrarily: there is no connection between the applied style and the content. Even empty paragraphs earned styles of different levels. On the other hand, the formats of the built-in styles are modified: the same levels got different formats (e.g. H7 style is applied with different formats for each instance). It is obvious that the author of the sample of Figure 7 did not read or did not understand the extract from "Add a heading" [36] but used the styles at the hyperattention phase.On the contrary, Figure 8 is a sample of using only one custom style everywhere in the document, which is another form of hyper attention mode,

3 na "Information on the Hungarian Higher Education System (modified in May 2015)". Retrieved February 24, 2019 from https://www.oktatas.hu/pub_bin/dload/ kepesitesek_elismertetese/DS_ENG_FINAL_2015_May.docx. 
the lack of design, deep attention. It is obvious from the extract that this document is part of a more complex one, which requires a Table of Contents. With these formats creating a Table of Contents of high-operational-use is impossible. Furthermore, the document runs with manual numbering (Chapter 3.2), which further lowers the level of operational use [18]. Both examples demonstrate that without activating the deep-attention phase, only low-operational-use documents can be created, with all its consequences.

\subsection{Manual Numbering}

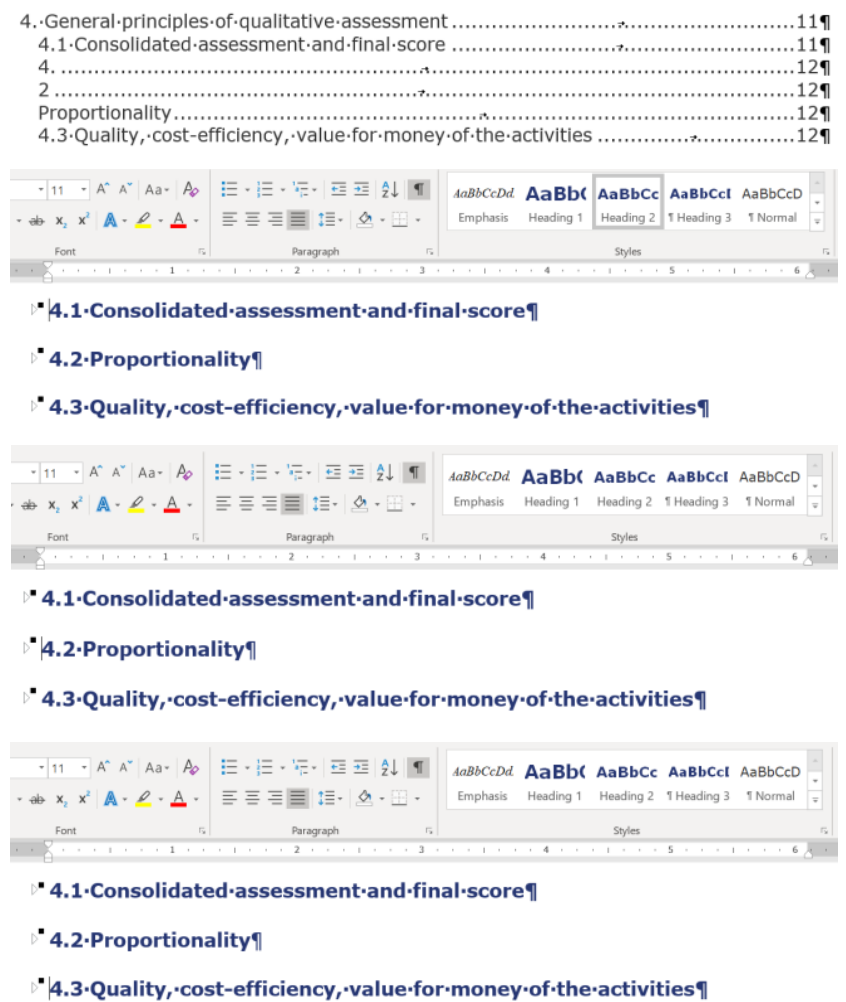

Figure 9

An incorrect use of built-in style which leads to incorrect automated Table of contents. ${ }^{4}$

Another form of distraction is, when the heading style is modified, and the Table of Contents cannot be created based on the assumed style (Figure 9). We also

4 na "Information on the Hungarian Higher Education System (modified in May 2015)". Retrieved February 24, 2019 from https://www.oktatas.hu/pub_bin/dload/ kepesitesek_elismertetese/DS_ENG_FINAL_2015_May.docx. 
must note that all the selected examples consist of manual numbering. As such, the numbering is not part of the formatted session and the style, consequently, is handled as any other character string of the text. Manual numbering, beyond not being part of the style, has the usual consequence of low-operational-use, meaning in this case that if any modification is made in the numbering, all the consecutive, connected sessions must be renumbered, which is a low-mathability approach to the problem.

\subsection{Built-in Presentation Styles}

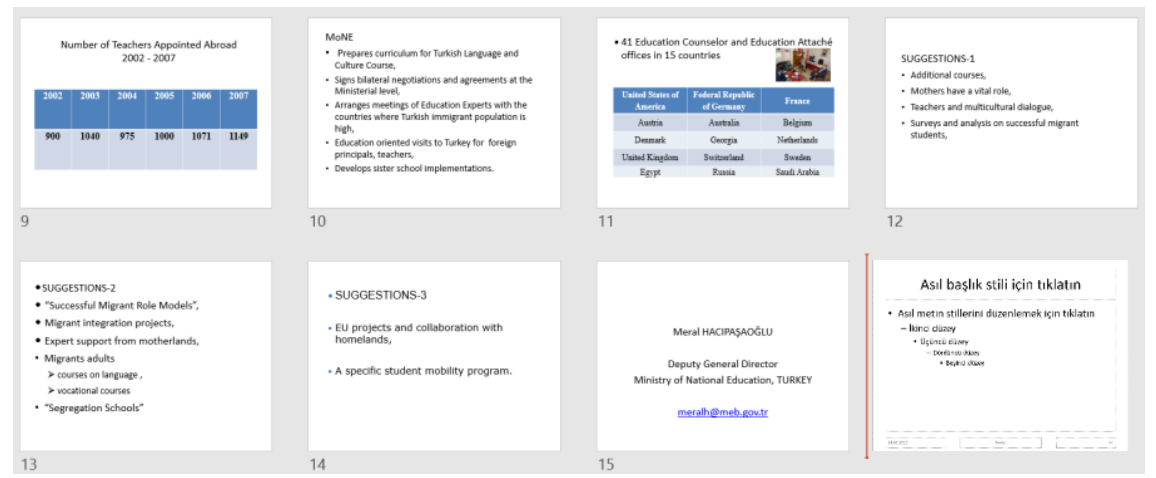

Figure 10

Various contents using the same Title and Content layout. The comparison of the slides with the Slide Master of Title and Content layout (lower right corner) clearly reveals that not only the selection of layouts is incorrect but also the use of the layout. ${ }^{5}$

Designing a presentation follows the same concept-based problem-solving method as detailed above. Analyzing presentations reveals that we are faced with the same problem: end-users only apply the third step of problem-solving, without giving a thought to design and discussion. All this happens, despite the effort of Microsoft. In PowerPoint, similar to Word, they set up layouts for various contents in the hope of convincing end-users to apply them. The documents found on the Internet clearly reveal that end-users, in general, are so hyper-attention-addicted that they do not even recognize when such obvious help is offered.

One of the most frequently met style errors in presentations is that regardless of the content the same layout is applied to the slides (Figure 10). Another form of distraction is when a selected style is misused. In the sample of Figure 10 the layout is completely ignored. In Figure 12 the background is set up in the Slide

5 M. Haciapsaqlou "Turkish Migrants and Education" Ministry of National Education, TURKEY. Retrieved February 24, 2019 from http://www.oecd.org/education/ innovation-education/41563670.ppt 
Master and applied correctly, however the paragraph and character formats of the layout are ignored on the slides. All the slides are formatted individually, manually, not leaving space for the utilization of styles, this again is a lowmathability solution.

\begin{tabular}{|c|c|c|}
\hline $\begin{array}{c}\text { United States of } \\
\text { America }\end{array}$ & $\begin{array}{c}\text { Federal Republic } \\
\text { of Germany }\end{array}$ & France \\
\hline Austria & Australia & Belgium \\
\hline Denmark & Georgia & Netherlands \\
\hline United Kingdom & Switzerland & Sweden \\
\hline Egypt & Russia & Saudi Arabia \\
\hline
\end{tabular}

Figure 11

Another form of ignoring style is presented in the table (Figure 10, Slide 11). Instead of using a list, a table style with a header row is applied, suggesting that the first row is the title. However, analyzing the content it is revealed that all the countries listed in the table play the same role, consequently, the selection of this style is not correct.

\subsection{Considering the Target Group}

One further aspect of planning presentations is the amount of content. This point of view plays a crucial role in any text-based document forms, however, presentations are the worst affected. It is a common practice that presentations hold the undesired amount of content, not considering the perceptive cognitive load of the audience.

To find a balance between the content and its presentation form requires a concept-based problem-solving approach by the author, which can only be carried out in deep-attention mode. On the other hand, the essence of the presentation is that it requires a hyper-attention mode from the audience. This paradox and the difficulty to resolve it can be an explanation to frequently encountered poorly designed presentations.

This paradox - creating in the deep attention phase by the author $\rightarrow$ perceiving in the hyper attention phase by the audience - weights a huge cognitive load on the authors, of which they usually are not aware of. 


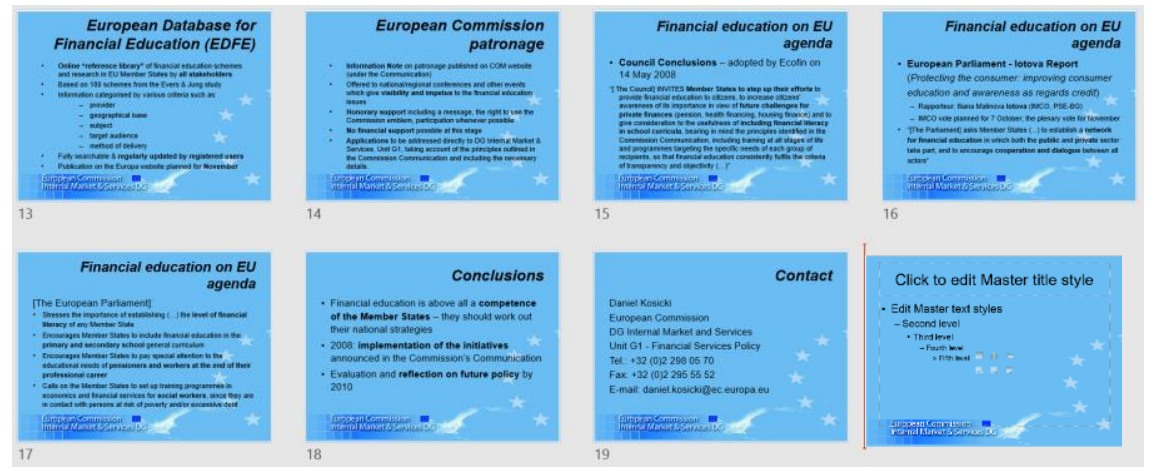

Figure 12

Slides with Title and Content layout seem to use the same formats. The comparison of the formats of the slides and the Slide Master of the Title and Content layout (lower right corner) reveals that the background is set up in the Slide Master, however, the paragraph and characters formats of the Slide Master are completely ignored and all the slides formatted individually. ${ }^{6}$

\section{Conclusions}

The present paper details how the different attention and thinking modes are connected to high- and low-mathability 'end-user computer problem-solving approaches'. Our investigation applies the HY-DE model framework, with its forms on the hyper and deep attention alterations and ratios. According to this model, the shift from hyper to deep attention requires necessary, well-designed teacher guidance through a mixed-phase of the two attention types.

- "I can do it, that's enough." - was our primary concern. However, the paper reveals that we must reformulate the slogan, and ask the question - How we can do it well. On the one hand, expressing that there is a difference between doing and doing well, on the other, we cannot do it alone, proper guidance from teachers are crucial for working effectively and efficiently.

The discussion is started with the presumed behavior of end-users, fulfilling the marketing slogans of software companies, and ends with the products of "real" end-users, wasting both human and computer resources, carrying out these outputs primarily guided by their ignorance. This ignorance is expressed in at least two forms: (1) the creation and modification of erroneous digital documents and (2) the dissemination of them. We searched for explanation and resolution to this paradox and found that the primary reason for the unexpected behavior of endusers is the selection of problem-solving methods. They prefer working with low-

6 D. Kosicki "European Commission initiatives for financial education" Magyar Nemzeti Bank. Conference on Financial Awareness. 2 October 2008, Budapest. Retrieved February 24, 2019 from http://www.oecd.org/education/innovationeducation/41563670.ppt. 
mathability approaches, surfing the interfaces of the programs in hyper attention mode without considering the specialties and details of the problems. With this tool-centered approach, end-users do not give thoughts to analysis, design, and discussion, in short, for deep attention problem-solving methods. One further concern of the topic is the selection of thinking modes. In previous works, it was found that thinking might be the explanation for this immense number of erroneous documents. However, we argue that deep attention mode cannot be left out, but should be applied along with hyper attention mode by finding the right proportion and situation, leaving also space for high-mathability computer problem-solving.

\section{References}

[1] P. Baranyi, A. Csapo A., \& G. Sallai: Cognitive Infocommunications (CogInfoCom), Springer International Publishing Switzerland, 2015, p. 191 (978-3-319- 19607-7, $\quad$ http://www.springer.com/us/book/ 9783319196077\#aboutBook

[2] P. Baranyi \& A. Csapo: Definition and Synergies of Cognitive Infocommunication, Acta Polytechnica Hungarica, 2012, Vol. 9, No. 1, pp. 67-83 (ISSN 1785-8860)

[3] P. Baranyi \& A. Gilányi: Mathability: Emulating and Enhancing Human Mathematical Capabilities, The $4^{\text {th }}$ IEEE International Conference on Cognitive Infocommunications, Budapest, Hungary, 2013, pp. 555-558, DOI=https://ieeexplore.ieee.org/document/6719309/

[4] TechTerms. End User, https://techterms.com/definition/enduser [13.13.2020.]

[5] Merriam-Webster. End user, https://www.merriam-webster.com/dictionary/ end\%20user [13.03.2020.]

[6] D. Kahneman: Thinking, Fast and Slow, New York, Farrar, Straus; Giroux, 2011

[7] E. Dani: The HY-DE Model: An Interdisciplinary Attempt to Deal with the Phenomenon of Hyperattention, Journal of Systemics, Cybernetics and Informatics, 2015, 13 (6), pp. 8-14

[8] K. Chmielewska and A. Gilanyi, "Educational context of mathability," Acta Polytechnica Hungarica, Vol. 15, No. 5, pp. 223-237, 2018

[9] K. Chmielwska and A. Gilányi, "Computer assisted activating methods in education," in $10^{\text {th }}$ IEEE International Conference on Cognitive Infocommunications (CogInfoCom). IEEE, 2019

[10] R. R. Panko: What We Know About Spreadsheet Errors, Journal of End User Computing. Special issue on Scaling Up End User Development, 2008, (10) 2, pp. 15-21 
[11] R. R. Panko \& S. Aurigemma: Revising the Panko-Halverson taxonomy of spreadsheet errors, Decision Support Systems, 2010, (49) 2, pp. 235-244

[12] R. R. Panko: The Cognitive Science of Spreadsheet Errors: Why Thinking is Bad, Proceedings of the $46^{\text {th }}$ Hawaii International Conference on System Sciences, Maui, Hawaii, 2013, pp. 1-10

[13] K. N. Hayles: Hyper and Deep Attention: The Generational Divide in Cognitive Modes [04.05.2018.]

[14] M. Csernoch \& E. Dani: Data-structure validator: an application of the HYDE model, The $8^{\text {th }}$ IEEE International Conference on Cognitive Infocommunications, Debrecen, Hungary, 2017, pp. 197-202

[15] P. Biró \& M. Csernoch: The mathability of computer problem solving approaches, The $6^{\text {th }}$ IEEE International Conference on Cognitive Infocommunications, Győr, Hungary, 2015, pp. 111-114, DOI=http://doi.org/10.1109/CogInfoCom.2015.7390574

[16] P. Biró \& M. Csernoch: The mathability of spreadsheet tools, The $6^{\text {th }}$ IEEE International Conference on Cognitive Infocommunications, Győr, Hungary, 2015, pp. 105-110, DOI= http://doi.org/10.1109/ CogInfoCom.2015.7390573

[17] M. Csernoch: Thinking Fast and Slow in Computer Problem Solving, Journal of Software Engineering and Applications, Vol. 10, No. 01 (2017), Article ID:73749, pp. 30 10.4236/jsea.2017.101002

[18] M. Csernoch: Do You Speak and Write in Informatics? $10^{\text {th }}$ International Multi-Conference on Complexity, Informatics and Cybernetics (IMCIC 2019), Orlando, Florida, USA 12-15 March 2019, pp. 157-152

[19] IEEE\&ACM Report. Computer Science Curricula 2013. The Joint Task Force on Computing Curricula Association for Computing Machinery (ACM) IEEE Computer

[20] J. B. Biggs \& K. E. Collis: Evaluating the Quality of Learning: The SOLO Taxonomy, 1982, Academic Press, New York

[21] J. M. Wing: Computational thinking, Communications of the ACM, 49(3) 2006, 33, DOI= http://doi.org/10.1145/1118178.1118215

[22] EuSpRIG, European Spreadsheet Risk Interest Group (2020), EuSpRIG Horror Stories. Retrieved 28 February 2020 from http://www.eusprig.org/ horror-stories.htm

[23] Gy. Pólya: How To Solve It. A New Aspect of Mathematical Method. Second edition, Princeton University Press, Princeton, New Jersey, 1945

[24] M. Prensky: Digital Natives, Digital Immigrants, From On the Horizon, MCB University Press, Vol. 9 No. 5, October 2001, http://www.marcprensky.com/writing/Prensky\%20- 
\%20Digital\%20Natives, \%20Digital\%20Immigrants\%20-\%20Part1.pdf [03.03.2018.]

[25] P. A. Kirschner, \& P. De Bruyckere: The myths of the digital native and the multitasker, Teaching and Teacher Education, 2017, 67, pp. 135-142

[26] J. Sweller, P. Ayres, \& S. Kalyuga: Cognitive Load Theory. Berlin: Springer, 2011

[27] M. Csernoch \& P. Biró: Edu-Edition Spreadsheet Competency Framework, Proceedings of the EuSpRIG 2017 Conference "Spreadsheet Risk Management", Imperial College, London, 2017, pp. 121-136

[28] P. L. Bewig: How do you know your spreadsheet is right? Principles, Techniques and Practice of Spreadsheet Style, 2005. https://arxiv.org/ftp/arxiv/papers/1301/1301.5878.pdf [12.11.2019.]

[29] Z. Przasnyski, L. Leon, \& K. C. Seal: In Search of a Taxonomy for Classifying Qualitative Spreadsheet Errors, Proceedings of EuSpRIG 2011 Conference, "Spreadsheet Governance - Policy and Practice", 2011

[30] National Research Council How People Learn: Brain, Mind, Experience, and School: Expanded Edition, National Academy Press, Washington, D.C., 2000

[31] C. Roda: Human Attention in Digital Environments, Cambridge University Press, 2011

[32] M. Ben-Ari: Bricolage Forever! PPIG 1999, 11 ${ }^{\text {th }}$ Annual Workshop, Computer-Based Learning Unit, University of Leeds, UK, 1999 http://www.ppig.org/papers/11th-benari.pdf [12.03.2015.]

[33] J. Kruger, \& D. Dunning: Unskilled and Unaware of It: How Difficulties in Recognizing One's Own Incompetence Lead to Inflated Self-Assessments, Journal of Personality and Social Psychology, 1999, 77 (6), pp. 1121-34

[34] R. Lister: After the Gold Rush: Toward Sustainable Scholarship in Computing, Proc. Tenth Australasian Computing Education Conference (ACE2008), Wollongong, Australia, CRPIT Volume 78 - Computing Education 2008

[35] N. Garrett: Textbooks for Responsible Data Analysis in Excel, Journal of Education for Business, 0: 1-6, 2015, Taylor and Francis Group, LLC, DOI: $10.1080 / 08832323.2015 .1007908$

[36] Microsoft, Add a heading, https://support.office.com/en-us/article/add-aheading-3eb8b917-56dc-4a17-891a-a026b2c790f2 [02.04.2019.] 